\title{
Retraction: Determinants of self-reported adherence to inhaler therapy in patients with chronic obstructive pulmonary disease
}

\author{
Ilaria Aredano, ${ }^{1}$ Francesca de Blasio, ${ }^{1}$ Paola Berchialla, ${ }^{2}$ Luisa Brussino, ${ }^{1}$ Caterina Bucca, ${ }^{1}$ Paolo Solidoro ${ }^{3}$ \\ ${ }^{1}$ Department of Medical Sciences, University of Turin \\ ${ }^{2}$ Department of Clinical and Biological Sciences, University of Turin \\ ${ }^{3}$ S.C. Pneumologia U, Respiratory Clinic Città della Salute e della Scienza, Turin, Italy
}

\begin{abstract}
On behalf of the coauthors and with much regret, I must retract our publication entitled "Determinants of self-reported adherence to inhaler therapy in patients with chronic obstructive pulmonary disease", published in Multidisciplinary Respiratory Medicine 2020;15:654.

The reason for the retraction is a complaint by MMAS Research LLC because of the breaching of the IRS Morisky Widget License.
\end{abstract}

Prof. Caterina Bucca

Retired Professor of Respiratory Medicine

Dept. Medical Sciences

University of Turin

Italy

Correspondence: Prof. Caterina Bucca, Department of Medical Sciences, University of Turin, corso Dogliotti 14 , 10126 Turin, Italy. E-mail: caterina.bucca@unito.it 